\title{
LA DEPRESIÓN, EVOLUCIÓN DEL CONCEPTO DESDE LA MELANCOLÍA HASTA LA DEPRESIÓN COMO ENFERMEDAD FÍSICA
}

\section{DEPRESSION, EVOLUTION OF THE CONCEPT FROM MELANCHOLY TO DEPRESSION AS PHYSICAL ILLNESS}

PATRICIO BENAVIDES E. ${ }^{1}$

\footnotetext{
${ }^{1}$ Pontificia Universidad Católica del Ecuador, Facultad de Psicología, Quito, Ecuador (jpbenavides@puce.edu.ec).
} 



\section{LA DEPRESIÓN, EVOLUCIÓN DEL CONCEPTO DESDE LA MELANCOLÎA HASTA LA DEPRESIÓN COMO ENFERMEDAD FÍSICA}

\section{DEPRESSION, EVOLUTION OF THE CONCEPT FROM MELANCHOLY TO DEPRESSION AS PHYSICAL ILLNESS}

Patricio Benavides E.

Palabras Clave: tristeza, depresión, trastornos afectivos, psicopatología, neuroplasticidad.

Key words: sadness, depression, affective disorders, psychopathology, neuroplasticity.

\section{RESUMEN}

En el presente artículo se hace un acercamiento a los aspectos conceptuales e históricos de la depresión y sus trastornos asociados; se establecen la diferencias entre los distintos conceptos con que se ha abordado su nosología y se revisan las aportaciones de las últimas épocas que han obligado a una redefinición del concepto de depresión que partiendo desde la descripción clásica de la melancolía, evoluciona hasta tomar en cuenta los distintos métodos de investigación biológica válidos y objetivos que permiten su mejor comprensión.

Estos métodos involucran sistemas neuro-anatómicos, endócrinos, inmunitarios, celulares, moleculares y genéticos, sin descuidar de ninguna manera la participación de la epigénética, que son los que nos llevan a una 
aceptación nueva de un amplio espectro de trastornos afectivos. Estos conocimientos son útiles porque facilitan la comprensión clínica y el abordaje terapéutico, además de permitir adaptar las diferentes modalidades de psicoterapia a los distintos tipos de trastornos y de pacientes depresivos. Concluyendo que todavía no se ha alcanzado un cuerpo sólido de conocimiento que logre una alta eficacia de los tratamientos, tanto a nivel psicológico como a nivel molecular.

\section{ABSTRACT}

The present article is an approach to the conceptual and historical aspects of the depression and its associate disorders; stablishing the differences between the distinct concepts with which its nosology has been approached revising the contributions of last epochs that have forced a redefinition of the concept of depression that setting off from the classic description of melancholy, evolves onto taking into consideration the distinct methods of biological research, valid and objective, that allow a better understanding. These methods involve neuro-anatomical systems, endocrinal, immune, cellular, molecular and genetic, by no means neglecting the participation of the epigenetics, which they are the ones that take us to a new acceptance of a wide spectrum of affective disorders. This knowledge is useful because it facilitates the clinical comprehension and the therapeutic approach, in addition to allowing the adaptation of different modalities of psychotherapy to the distinct types of disorders and of depressive patients. Concluding that there has not yet been reached a solid body of knowledge to achieve a high treatment, at a psychological level as at a molecular one.

\section{INTRODUCCIÓN}

Los conocimientos actuales que se tienen sobre la depresión, son muy heterogéneos, las distintas disciplinas que se encargan de su abordaje se han nutrido de modelos o diferentes concepciones teóricas que se han dado a lo largo de la historia. La nosología, la psicopatología, la comprensión de los procesos del enfermar psíquico han alcanzado un grado enorme de desarrollo con la ayuda de las Neurociencias y de sus disciplinas conexas como la Neuro- 
genética, la Psicofarmacología, las técnicas de inmunología, de neuroimagen, etc.; y es por eso que a la hora de definir el concepto actual de depresión, se tiene que tomar en consideración el contexto de los diferentes modelos presentes actualmente en psiquiatría y psicología.

En este artículo se resaltan los aportes a esta comprensión del concepto de depresión desde sus orígenes, hasta los últimos estudios que dan cuenta de los cambios que suceden en el sistema nervioso y en el propio organismo cuando se presenta la depresión. Se justifica el concepto que la depresión ha tenido una extraordinaria plasticidad clínica ante las contingencias socio-culturales (Alonso Fernández, 1979), el estar deprimido, no es solo un estado psíquico sino una alteración física donde el sujeto no solo se siente triste, sino que además y fundamentalmente, "se siente enfermo"

\section{TRISTEZA, MELANCOLÎA, DEPRESIÓN, EVOLUCIÓN DEL CONCEPTO}

La tristeza es un sentimiento universal, consustancial con la existencia del hombre, forma parte de las funciones afectivas o también conocidas como funciones del humor y su presentación obedece a una respuesta a las adversidades de la vida del sujeto. La tristeza sin causa, sin motivo es la que no guarda relación con los factores desencadenantes, y en caso que los hubiera, no tienen proporcionalidad con la intensidad, la duración y la funcionalidad del individuo. A esta última se la conoce como tristeza patológica, o mejor con el término de "depresión". Sin embargo, hay que reconocer que este término de depresión ha sido objeto de un mal uso y de un abuso, tanto por la gente común como por los profesionales y estudiosos de este fenó- meno en donde psicólogos y psiquiatras han sido cómplices de este mal uso al psicologizar hechos o respuestas normales de la esfera afectiva.

Esta alteración patológica del humor, en realidad es un grupo heterogéneo de enfermedades o trastornos afectivos, con un espectro muy amplio que incluyen cuadros clásicos como la "depresión mayor" hasta cuadros atípicos, secundarios o variantes de una forma de reacción psíquica normal; pero, que comparten como característica de este humor patológico, la extremada disfuncionalidad o limitación de sus capacidades, la cronicidad del mismo y el acompañamiento de otros síntomas propios de la depresión.

En la conceptualización de la depresión y de los trastornos afectivos, ha 
variado la expresión sintomatológica, clave para entender la delimitación clínica y diferenciar la tristeza, propia de la esfera de las emociones normales del ser humano, de la tristeza patológica o depresión. Varios trastornos considerados distintos de los afectivos, como las neurosis, trastornos conductuales y algunas alteraciones psicosomáticas, han sido reconsideradas por los estudios psicopatológicos de grandes maestros como López-lbor, Schneider, Weitbrech, Tellebanch y otros (Chinchilla Moreno, 2011)

Es distinto hablar de estado de ánimo y de sentimientos. Como estado de ánimo o humor se conoce a un estado afectivo de carácter relativamente autónomo y duradero en el tiempo que repercute en las experiencias psicológicas y corporales del sujeto. Los sentimientos son vivencias muy variables, más de naturaleza psicológica como la pena, la alegría, la ira. Sentimientos y humor son fenómenos independientes pero interactuantes. El estado de ánimo y los sentimientos se expresan de manera diferente en el tiempo. Los estados de ánimo son duraderos, persistentes, estables. Los sentimientos son breves. Las alteraciones del estado de ánimo son relativamente autónomas del ambiente y muchas veces comienzan $y$ terminan sin motivo aparente, mientras que los sentimientos están unidos a las circunstancias vitales y tienen un comienzo y final paroxístico (Peralta y Cuesta, 2002). El estado de ánimo se relaciona con la personalidad, es involuntario, no está mediado por procesos psicológicos. Los sentimientos son reactivos, dirigidos intencionalmente, son fenómenos sociales.

Soria (2011) atribuye a la depresión distintas connotaciones; la depresión se presenta como un síntoma único, alguna vez puede acompañarse de otros síntomas no relacionados; la depresión puede presentarse como un síndrome en el que lo nuclear es la tristeza a la que le acompañan otros síntomas que le relacionan con un trastorno afectivo, o bien la depresión puede ser una enfermedad, así mismo que tiene como síntoma característico la tristeza y que cuenta como cualquier otra con una etiología, una patofisiología, un curso, una evolución y una terapéutica propias.

La evolución del concepto de depresión contempla varios factores incluidos los culturales y los neurobiológicos, sobre todo estos, en las últimas décadas adquieren especial relevancia puesto que contribuyen con las evidencias para su sistematización, su comprensión clínica, su clasificación, su prevención y tratamiento (Chinchilla Moreno, 2011).

Las primeras menciones hacen referencia a la descripción de Areteo de Capadocia, quien en el siglo I de nuestra era es uno de los primeros en describir 
la melancolía y la manía como los extremos de una misma enfermedad, en la una caracteriza la tristeza y el abatimiento, mientras que en la otra la ira y el regocijo. Más tarde sería corroborada por los clínicos islámicos y occidentales y hasta por el propio Galeno quien perfeccionó estos conocimientos con la descripción de la melancolía, la misma que resultaba de un exceso de bilis negra. La melancolía es un tipo de locura crónica, sin fiebre, propia de varones de edad madura en la que el paciente se presentaba cansado, triste, abatido, taciturno, con deseos de morir. A los síntomas psicológicos se podían sumar síntomas gastrointestinales como dolor abdominal, flatulencia y eructos que se localizaban en el estómago y el diafragma, área conocida como hipocondrio, de donde proviene justamente el término de hipocondría que simboliza a esos enfermos melancólicos con preocupaciones excesivas sobre sus síntomas corporales. Pero fue Hipócrates quien relacionó con más precisión y basado en su teoría humoral la descripción de los cuatro temperamentos básicos y su relación con la salud - enfermedad, en los que el melancólico resultaba tener un predominio de bilis negra a diferencia del sanguíneo (sangre), del bilioso (bilis amarilla) y del flemático (flema). Este tipo de pensamiento sobre el desequilibrio de los humores permaneció vigente hasta los siglos XV y XVII en que se establecen nuevas concepciones para la melancolía.

En la época medieval, la filosofía y la religión aportaron a su definición. Para la filosofía, la melancolía se trataba de una "alienación mental que nada tenía que ver con el cuerpo, y que incapacitaba para el ejercicio de las funciones racionales" mientras que el pensamiento mágico religioso volvió a imponerse para explicar los trastornos mentales como hechos sobrenaturales y endemoniados, en que la melancolía era propia de los poseídos, hechizados y embrujados; para la religión se trataba de "una repulsa moral", consecuencia, mediante la bilis negra, del recuerdo del pecado o de la tristeza subsiguiente al acto pecaminoso (Chinchilla Moreno, 2011). La melancolía tenía entonces, una concepción estrictamente moral que reñía con la experiencia cristiana alienada del enfermo.

No está bien claro a quien se le atribuye el término de depresión, al parecer el primero en mencionar es Richard Blackmore en 1725 que describe al Rey Guillermo III de Inglaterra, de estar deprimido en una profunda tristeza y melancolía. Durante el renacimiento, se desplaza la concepción mágico moralista y crece un enfoque más médico y humano de los enfermos mentales. Con la implementación de los estudios anatómicos, se invalida la teoría humoral y se promueve una nueva perspectiva para la 
comprensión de la depresión. Se introduce la posibilidad de la intervención de factores desencadenantes y se habla de melancolías reactivas.

En el siglo XVII Thomas Willis propone otros principios diferentes a los humores, que se basa en el hecho que unos espíritus (tierra, agua, sal, etc.) interaccionan entre sí de manera que el líquido nervioso que circula en el cuerpo, y que normalmente es benigno y sutil, se transforma en ácido y corrosivo. La circulación se ralentiza, la sangre se acumula en la zona precordial y se reduce en la cabeza y extremidades, el alma se debilita, el cuerpo se debilita y tiembla, por eso se expresan la tristeza y el miedo como síntomas nucleares de la melancolía (Cobo, 2005).

En los siglos XVIII y XIX, surgen las descripciones psicopatológicas de la melancolía. Pinel y Esquirol diferencian los trastornos con delirios únicos, exclusivos de la melancolía, de los delirios generales, extravagantes y furiosos que caracterizan a otras alteraciones de carácter cíclico como las psicosis maniacas. Kraepelin valida los conceptos de psicosis introducido por Von Feuchtersleben y el de endógeno, de Moebius (1983), así como también empieza a usar el término de depresión en lugar del clásico de melancolía; desde entonces se generaliza su uso en los clínicos. Kraepelin establece criterios para diferenciar los distintos tras- tornos mentales; por ejemplo el criterio etiológico, con el que describía a ciertos trastornos en los que se detectaban alteraciones anatomofisiológicas propias en los cuadros descritos por Alzheimer y Korsakov de otros donde no estaba seguro que estos se presentaban como en las depresiones. También describió un elemento diferenciador llamado pronóstico, que le permitía distinguir las alteraciones mentales curables como la melancolía, la manía, el delirio agudo, de las alteraciones incurables como la locura periódica circular y el delirio crónico; propone el término de psicosis maniaco depresiva para estas últimas (Cobo, 2005).

En la escuela psicodinámica, Freud compara a la melancolía con estados similares al duelo o aflicción; condiciones necesarias de un cambio interno por la pérdida real o imaginaria del objeto deseado. Freud argumenta que la depresión endógena se manifiesta como "una respuesta de malhumor, displacer y pérdida de interés por el entorno, con una incapacidad para amar, una merma en autoestima que le conduce a los autorreproches con riesgo de autocastigos suicidas, con una regresión narcisista, sugiriendo la pérdida objetal por procesos intrapsíquicos personales desviados" (Chinchilla Moreno, 2011).

Se da entonces un cambio fundamental en el abordaje de los trastornos afectivos y especialmente de la depre- 
sión. Desde la hegemonía alcanzada por la psiquiatría anglosajona en general y la norteamericana en particular a final del siglo pasado, que quedó entrampada por décadas en una concepción casi exclusivamente psicodinámica del enfermar psíquico; lo cualitativo ha perdido protagonismo para cederlo a lo dimensional. Se da paso a las clasificaciones por consenso que han venido a sustituir a las descripciones de grandes psicopatólogos que describieron con una riqueza hasta ahora sin parangón los fenómenos que subyacen a los síntomas que se presentan en los trastornos afectivos. Hoy se ha dado prioridad a sistemas clasificatorios de las enfermedades mentales donde se enfatizan las presentaciones sintomáticas, el curso, el tiempo de evolución, etc., en desmedro de la riqueza psicopatológica que acompañaba al diagnóstico.

Tal como se ha anotado anteriormente, las características de la depresión melancólica no han sido del todo bien definidas, pese a que todas las descripciones coinciden en síntomas nucleares como la tristeza, la desidia, el desgano de vivir, esto ha dado lugar a que en la actualidad se haya establecido que las depresiones constituyen un grupo heterogéneo de trastornos afectivos, frente a los cuales algunos autores consideran que se trata de entidades distintas entidades, cada una de ellas con sus etio- logía, curso, evolución y tratamientos propios, a lo que se denomina "criterio categorial", donde la melancolía, la depresión reactiva, la depresión mayor serían un ejemplo de ello; mientras que para otros autores, que tienen un "criterio dimensional" la depresión es un fenómeno "continuum", donde en el un extremo del grado de expresión psicopatológica estaría la melancolía como la expresión más leve y en el otro estaría la depresión mayor o endógena como la más grave.

Cuando a la depresión se le adiciona el calificativo de endógeno, se le relaciona con factores genéticos como agentes desencadenantes, mientras que el de exógeno se le relaciona con la génesis dada por los factores ambientales; aunque no necesariamente, ya que la sola condición de endogenicidad, no es suficiente como para que se dé el cuadro afectivo. Si la constitución hereditaria es muy fuerte es probable que se exprese el trastorno sin que haya estresores ambientales; pero así mismo, hechos vitales psico-traumatizantes fuertes y repetidos pueden romper un precario equilibrio vital de fondo genético poco evidente. A esto se puede sumar la presencia de factores somáticos, factores de personalidad y factores situacionales graves, intercurrentes (Chinchilla Moreno, 2011).

Por lo tanto, en la etiología de los trastornos afectivos se deben tomar en consideración factores biológicos, 
psicológicos y sociales, y en determinados casos uno de ellos será el más preponderante. Se dio un gran paso a la comprensión de la depresión con el advenimiento de las teorías biológicas que centraban su fisiopatología en el nivel de concentración de los neurotransmisores y sus metabolitos o precursores. Luego la atención se centró en los receptores. Ahora el interés se centra en los procesos intracelulares y los cambios relacionados con el cur- so de la enfermedad y el tratamiento psicofarmacológico. Se trata de hallar un conjunto de biomarcadores indicadores de depresión o de otros trastornos mentales y de su posibilidad de predicción de respuestas terapéuticas, centrados en las características celulares, neuroquímicas, neuroendócrinas, genéticas y neuroinmunológicas, que se pudieran medir simplemente en sangre periférica.

\section{CAMBIOS NEUROPLÁSTICOS}

La presentación de la depresión no es solo una manifestación de la esfera psíquica, sino que se acompaña de una serie de mecanismos patogénicos que le convierten a esta en una enfermedad física como tal y con el consiguiente riesgo de desarrollar enfermedades médicas e incrementar la mortalidad. El padecer psíquico se ha transmutado en "sufrimiento corporal". De allí que el paciente depresivo en su concepción no es solo el que se siente "triste" sino el que sabe que se siente "enfermo" o inadecuado en su adaptación. Los síntomas son más que los signos. (Soria, 2011) En la concepción clásica de la depresión el núcleo de su manifestación era el "trastorno del humor", en la actualidad, el núcleo principal es "el trastorno del hacer", demostrable cuando el paciente se queja de que "Io cotidiano se le vuelve extraordinario".

\section{DEPRESIÓN Y SISTEMA INMUNE}

Desde los años 60 ya se ha probado, aunque no de manera concluyente, la participación de los factores estresantes en el aparecimiento de trastornos en el sistema inmunológico, que favorecen el aparecimiento de enfermedades infecciosas, enfermedades autoinmunes, enfermedades cardiovasculares, diabetes y 
el cáncer. El diagnóstico de diabetes duplica la posibilidad de sufrir depresión. El $70 \%$ de los pacientes con enfermedades autoinmunes experimentan depresión. 15 - $25 \%$ de pacientes con cáncer experimentan depresión, en relación con el $9 \%$ en la población general. Así mismo se ha evidenciado que el sistema inmunológico es capaz de modular la actividad de los neurotransmisores y del sistema endócrino; en este último lo hace a través de cambios en el eje hipotálamo-hipofiso-adrenal, provocando aumento en la liberación de citoquinas, del Factor Liberador de Corticotrofina (CRF), de la Hormona Liberadora de Tirotrofina; del Factor Liberador de la Hormona de crecimiento y de la Somatostatina. En los estudios de neuroimagen, se observan que el aumento en la secreción de CRF produce un aumento del volumen de la hipófisis y de las suprarrenales y el exceso de glucocorticoides resultante llega a dañar estructuras cerebrales como el hipocampo, lo cual se puede relacionar con las alteraciones cognitivas de la depresión. Es por eso que en la clínica se observan que pacientes deprimidos severos y hasta con intentos de suicidio tienen mayores concentraciones de cortisol en plasma, orina y líquido céfalo raquídeo; sin embargo, la mayoría de estos estudios han sido en adultos y muy pocos en adultos mayores. (Rhebergen, y otros 2015). En estos pacientes, el test de supresión de dexametasona, la supresión de adrenocorticotropina y cortisol están alterados y se normalizan cuando disminuyen los síntomas depresivos luego del tratamiento. Bien puede usarse este test como un marcador biológico para evidenciar la respuesta y la mejoría a la terapia antidepresiva (Navas, 2009).

Pese a que en sujetos deprimidos se produce un aumento en la producción de cortisol, este no funciona de manera adecuada, y se relaciona con una disminución de la actividad de los receptores de glucocorticoides.

Cuando hay un proceso inflamatorio el cuerpo libera citoquinas, compuestos proteicos responsables de señales celulares que activan al sistema inmunitario. Estas son citoquinas tipo 1 y tipo 2, interleucinas, todas ellas encargadas de activar las respuestas celulares y de anticuerpos para combatir la inflamación. El aumento considerable de estos marcadores inflamatorios se asocia con la depresión (De los Ángeles, 2014). Existen varios estudios en animales tratados con antidepresivos que demuestran que se produce una normalización del sistema inmunológico disminuyendo sobre todo la producción de citoquinas proinflamatorias y de aumento de la producción de citoquinas antinflamatorias como la interleuquina-10, IL-10 con la consiguiente disminución de los procesos inflamatorios en el cerebro, 
de una atenuación de la hiperactividad del eje hipotálamo-hipofiso-adrenal y de normalización de la actividad de los receptores de los glucocorticoides en el hipocampo (Navas, 2009). Hoy se está investigando si el tratamiento con antinflamatorios puede mejorar los síntomas depresivos, o si estos pueden aumentar los efectos de los tratamientos antidepresivos. La administración de altas dosis de citoquinas proinflamatorias puede inducir síntomas depresivos y la atenuación de estas citoquinas con antinflamatorios pueden reducir los síntomas depresivos (Pollak Y., 2002). Los estudios no son consistentes y no dan cuenta de esta relación en todos los casos, lo que lleva a pensar en un subtipo inflamatorio de la depresión (De los Ángeles, 2014).

Los estudios que revelan la existencia de elevadas cantidades de mediadores inflamatorios tipo interleucinas o factores alfa de necrosis tumoral, les asocian a una disregulación del tono autonómico con la consiguiente producción de trastornos cardiovasculares, con la progresión de la enfermedad coronaria y la disregulación de la glucosa, de allí la asociación depresión - diabetes y síndrome metabólico (Mezuc, 2008).

\section{DISREGULACIÓN DEL EJE HIPOTÁLAMO HIPOFISARIO}

Se ha demostrado que las anormalidades del eje hipotálamo-hipofisoadrenal se transmiten genéticamente, al encontrar casos de sujetos sanos pero con historia familiar de trastornos afectivos. El eje hipotalámico-hipofiso-adrenal y de las monoaminas del sistema nervioso autónomo es modular la función inmunológica. Las altas concentraciones de corticoides adrenales como el cortisol, tienen un profundo efecto supresivo sobre el Sistema Inmune, aunque en baja cantidad pueden tener un pequeño efecto estimulante. Los estímulos tanto fisiológicos como psicológicos activan el eje HPA a través de la activación córtico límbica, hecho que resulta en la liberación de CRF, ACTH y de cortisol plasmático. Cuando el nivel de cortisol se eleva hay una larga serie de funciones inmunes que se suprimen. Luego se produce un efecto de feedback inhibitorio del cortisol a nivel hipotalámico e hipofisario que reduce la secreción de CRF (Bonet, 2011) (Irwin, 1995). 


\section{DEPRESIÓN Y DISREGULACIÓN DE MECANISMOS DE CONTROL CELULAR}

El factor neurotrófico derivado del cerebro, BDNF (inglés), es un factor de crecimiento que regula funciones cruciales en el sistema nervioso central, como la supervivencia celular, la diferenciación celular, el crecimiento axonal y dendrítico, contribuyendo de esta manera con la plasticidad (Bettina $\mathrm{H}$. Bewernick y Thomas E. Schlaepfer, 2013). En una revisión sistemática de varios metanálisis sobre los niveles de BDNF en sangre y su correlación con la neuroplasticidad en la depresión, Rusowski, Lopes y Fregni (2008) comparan pacientes con niveles bajos de BDNF y pacientes antes y después del tratamiento con antidepresivos y observan que los niveles de BDNF están asociados a los cambios clínicos de la depresión, y que la mejoría de la depresión se asocia con cambios en la neuroplasticidad después de los tratamientos con antidepresivos.

El BDNF es una neurotrofina que se relaciona con la supervivencia neuronal, las señales sinápticas y la consolidación sináptica (Rusowski, Lopes \& Fregni, 2008). No se ha establecido mediante la teoría monoaminérgica, una causalidad directa de la depresión ni tampoco la depresión se explica solo por los niveles bajos de monoaminas. Se ha relacionado una disminución del tamaño del hi- pocampo y de la amígdala asociadas a la depresión y a una disfunción del eje hipotálamo hipofiso adrenal, traducidos en un déficit de memoria y deterioro cognitivo, que son reversibles con la terapia antidepresiva, lo cual se evidencia con estudios de neuroimagen y de incremento de los puntajes en las escalas de depresión (Friedman, 2008).

Queda la evidencia que la tristeza es una emoción habitual y que tiene unos límites difusos e imprecisos con la conceptualización de la depresión; la depresión puede ir desde una reacción normal frente a acontecimientos adversos de la vida o puede presentarse como un síntoma, un síndrome o una enfermedad. El término de "depresión"es muy genérico e inespecífico y engloba a un grupo heterogéneo de entidades clínicas. El término de "trastorno depresivo" da cuenta que hay una condición clínica más relevante y están involucrados más factores ambientales, mientras que el término "enfermedad depresiva" se reserva para casos más graves, con síntomas endogenomorfos y de carácter más biológico que ambiental (Peralta y Cuesta, 2002).

Mucho se ha discutido sobre la necesidad del tratamiento de la depresión, se sabe que la evolución natural de 
un episodio depresivo es hacia la resolución espontánea en un período que va de los tres a los veinticuatro meses; lo cual indicaría que no hace falta un tratamiento; sin embargo, más del $75 \%$ de pacientes depresivos vuelven a presentar una recaída en los meses siguientes, ya que por definición la depresión es una enfermedad cíclica, recurrente con períodos asintomáticos de duración variable. (Wikinski y Jufe, 2006). La discusión también se ha centrado en si el tratamiento de la depresión debe realizarse con psicoterapia o farmacoterapia. Estudios demuestran que la psicoterapia es efectiva si la depresión es leve, sobre todo en pacientes con baja tasa de recurrencia. (Gartlehner, y otros, 2015). Estos autores realizan una investigación exhaustiva al revisar varios estudios en donde se compara el uso de antidepresivos con terapias psicológicas y otras terapias alternativas, observando que no hay diferencia significativa entre ellas cuando se trata de depresión leve; en las depresiones moderadas observaron que la combinación de antidepresivos con acupuntura o antidepresivos con psicoterapia interpersonal tenía mejores resultados que el uso solo de antidepresivos, aunque tampoco demostraron diferencias significativas. Así mismo la Asociación Americana de Psicología recomienda el uso de distintas modalidades de psicoterapia, argumentando que los beneficios son significativos, (Cuijpers, Andersons y Straters, 2008) aunque reconoce que los estudios no son concluyentes. En los casos de depresiones moderadas o graves, la evidencia recomienda que se debe usar una combinación de antidepresivos más psicoterapia. (Atención integral de los adultos con diagnóstico de episodio depresivo, 2013) La psicoterapia más indicada es la terapia cognitiva conductual seguida de la psicoterapia interpersonal. (Cuijpers P., Straten A., y Schaik, 2009) El uso de la psicoterapia psicodinámica tiene una evidencia débil. (Simpson y otros, 2003) 


\section{CONCLUSIONES}

El concepto de depresión ha sufrido cambios en la clínica psicológica y psiquiátrica que han permitido en la actualidad, una mayor comprensión de su patogenia, su diagnóstico, su abordaje terapéutico y hasta su prevención.

La depresión es una enfermedad funcional, reversible, recurrente y una de las más tratables, evolutivamente tiende a la cronicidad, se expresa por cambios estructurales que de no ser tratada puede llevar a cambios neurodegenerativos.

En resumen, se plantea que en la depresión existe una afectación concomitante de los sistemas inmunológicos, endócrinos y celulares del sistema nervioso, con aumento de la susceptibilidad a la inflamación, a los trastornos hormonales y a los cambios patoplásticos en el sistema nervioso, lo que hace pensar que la depresión se trata de una disregulación cuantitativa de mecanismos homeostáticos cerebrales, que a su vez son los responsables de los cambios somáticos en todo el organismo que se dan en la depresión, con la consecuente afectación de la participación reforzadora de la voluntad, donde las actividades mínimas como hablar, caminar, pensar, concentrarse son incapaces de realizarse, por eso el deprimido no solo que se siente triste sino que además y de ma- nera importante, se siente "enfermo". Esta afectación a su estado físico, le lleva a pensar de manera deliroide en la preocupación por la salud del cuerpo y el temor a la enfermedad; de esta manera se aclara el concepto que la "depresión es una enfermedad física."

Todos los cambios en estos sistemas integradores del organismo, le convierten a la depresión es una condición clínica que para quien la padece va a comprometer su salud física. A nivel mundial, el 70 \% de las depresiones cursan con síntomas físicos, especialmente de dolor, estos se asocian a síntomas cardiacos, gastrointestinales, músculo esqueléticos, etc, lo que provoca que el paciente acuda a la consulta por estas quejas y no por la depresión. En las consultas de atención primaria, el médico asocia estas quejas a otras enfermedades y deriva a otros profesionales y no al psicólogo o al psiquiatra. Se estima que un deprimido antes de ser evaluado por el psiquiatra, ha visitado antes por lo menos a tres médicos.

La afectación física de la corporalidad, produce un incremento sostenido de su morbilidad, un aumento elevado a padecer de otras enfermedades y a una afectación de la calidad de vida, ya que puede llegar inclusive a producir una dis- 
capacidad en varios ámbitos en los que solo un tratamiento adecuado puede modificar su curso y pronóstico.

Con todo lo que se ha expuesto anteriormente, se puede entender que se ha producido un cambio en la comprensión de la clínica psiquiátrica de la depresión; la tendencia anterior de ver a la depresión solo en una expresión estrictamente psicopatológica ha dado lugar ahora a una hipocondrización o somatización de la misma, es decir se ha dado paso a entender que en la depresión se produce un incremento significativo de la traducción somática del padecer psíquico, el padecer psíquico se ha transmutado en sufrimiento corporal, es cada vez más un trastorno del cuerpo, y no del cuerpo real, sino de la vivencia de la corporalidad. Ya no es la salud del alma la que preocupa, es el cuerpo el que se "enferma" 


\section{BIBLIOGRAFÎA}

«Atención integral de los adultos con diagnóstico de episodio depresivo.» Guía de atención integral para la detección temprana y diagnóstico del episodio depresivo y trastorno depresivo recurrente en adultos. Bogotá: Ministerio de Salud y Protección Social, República de Colombia., 2013.

Bettina H. Bewernick, MSc (Psych), PhD, y MD Thomas E. Schlaepfer. Dialogues in clinical Neurosciencie. 15 de 1 de 2013. http://www.dialogues-cns.com/publication/chronic-depression-as-a-model-disease-for-cerebral-aging/.

Bonet, J. «http://www.gador.com.ar/ iyd/psiquiatria/pdf/vulner.» 2 de agosto de 2011. file:///C//papi/ www/htdocs/iyd/vulner/bonet. htm[02/08/2011 03:58:48 p.m.].

Chinchilla Moreno, A. (2011). «Aspectos conceptuales e históricos de la depresión.» En La depresión y sus máscaras., de A. Chinchilla Moreno, 7 - 35. Buenos Aires: Médica Panamericana.

Cobo, J. «Psiquiatría.com.» 28 de noviembre de 2005. http://www. psiquiatria.com/depresion/ el-concepto-de-depresion-historia-definiciones-nosologia-clasificacion/\#.
Cuijpers P, P, A Straten A, y A Schaik. «Psychological treatment of depression in primary care: a meta-analysis.» Br J Gen Pract., 2009: 51-60.

Cuijpers, P, G Andersons, y J Straters. «Psychotherapy for depression in adults: a.» J Consult Clin Psychol, 2008: 909-992.

De los Ángeles, Carlos. Salud Mental y complejidad. 1 de octubre de 2014. http://smcomplejidad.com/introduccion-la-relacion-depresion-inflamacion/.

Friedman, J. Hurley, R. Taber, K. (2008). «Bipolar Disorder. Imaging State Versus trait.» En Windows to the brain. Insights from Neuroimaging, de R. Hurley, 67-74. Washington: American Psychiatric Publishing, Inc.

Gartlehner, Gerald, Bradley Gaynes, Amick Amick, y Gary Asher. «PubMed Health.» Dec de 2015. https://www.ncbi.nlm.nih.gov/ pubmedhealth/PMH0084297/.

Irwin, M. «lrwin M (1995) Psychoneuroimmunology of Depression.» Psychopharmacology: The Fourth Generation, 1995: 34-40.

Mezuc, y cols. «Depression and type 2 diabetes over the livespan: a meta-analyses.» Diabetes Care, 2008: 
$2383-2390$.

Navas, Carmen, \& Zapata, Deliana. «Aspectos inmunológicos en la depresión.» Revista Venezolana de Oncología, 21(4), 2009: 244 - 252.

Peralta, V., y M.J. Cuesta. «Psicopatología y clsificación de Iso trastornos depresivos.» Anales Sis San NAvarra, 2002: 7 - 20.

Pollak Y, Yirmiya R. Cytokine-induced changes in mood and behaviour: "Cytokine-induced changes in mood and behaviour:implications for 'depression due to a general medical condition',.» Int J Neuropsychopharmacol, 2002: $389-399$.

Rhebergen, D, N Korten, BW Penninx, y ML Stek. «Hypothalamic-pituitary-adrenal axis activity in older persons with and without a de- pressive disorder.» Psychoneuroendocrinology., 2015: 341 -50. Simpson, S, R Corney, P Fitzgerald, y J Beecham. «A randomized controlled trial to evaluate the effectiveness and cost-effectiveness of psychodynamic counselling for general practice patients with chronic depression.» Psychol Med, 2003: 229-239.

Soria, C. «Estrés.doc.» Epigenetica_Factores_Postnatales. S.f. http://www. ipnie.com/DesarrolloDePatologias/Epigenetica_Factores_Postnatales.pdf.

Wikinski, Silvia, y Gabriela Jufe . «Tratamiento farmacológico del trastorno depresivo mayor.» En El Tratamiento Farmacológico en Psiquiatría, 141-157. Buenos Aires: Panamericana, 2006. 